\title{
Antiallodynic Action of 1-(3-(9H-Carbazol-9-yl)-1-propyl)- 4-(2-methyoxyphenyl)-4-piperidinol (NNC05-2090), a Betaine/GABA Transporter Inhibitor
}

\author{
Ayako Jinzenji ${ }^{1,2}$, Chiharu Sogawa ${ }^{2}$, Takuya Miyawaki ${ }^{1}$, Xue-fang $\mathrm{Wen}^{2}$, Dan $\mathrm{Yi}^{2}$, Kazumi Ohyama ${ }^{3}$, \\ Shigeo Kitayama ${ }^{2}$, Norio Sogawa ${ }^{2}, *$, and Katsuya Morita ${ }^{4}$ \\ ${ }^{1}$ Department of Dental Anesthesiology and Special Care Dentistry, ${ }^{2}$ Department of Dental Pharmacology, \\ Okayama University Graduate School of Medicine, Dentistry and Pharmaceutical Sciences, \\ 2-5-1 Shikata-cho, Kita-ku, Okayama 700-8525, Japan \\ ${ }^{3}$ RI Research Center, Okayama University Dental School, 2-5-1 Shikata-cho, Kita-ku, Okayama 700-8525, Japan \\ ${ }^{4}$ Department of Pharmacology, Faculty of Nursing, Hiroshima Bunka Gakuen University, \\ 2-10-3 Agaminami, Kure-city, Hiroshima 737-0004, Japan
}

Received August 19, 2013; Accepted April 23, 2014

\begin{abstract}
The GABAergic system in the spinal cord has been shown to participate in neuropathic pain in various animal models. GABA transporters (GATs) play a role in controlling the synaptic clearance of GABA; however, their role in neuropathic pain remains unclear. In the present study, we compared the betaine/GABA transporter (BGT-1) with other GAT subtypes to determine its participation in neuropathic pain using a mouse model of sciatic nerve ligation. 1-(3-(9H-Carbazol-9-yl)-1-propyl)-4-(2-methyoxyphenyl)-4-piperidinol (NNC05-2090), an inhibitor that displays moderate selectivity for BGT-1, had an antiallodynic action on model mice treated through both intrathecally and intravenous administration routes. On the other hand, SKF89976A, a selective GAT-1 inhibitor, had a weak antiallodynic action, and (S)-SNAP5114, an inhibitor that displays selectivity for GAT-3, had no antiallodynic action. Systemic analysis of these compounds on GABA uptake in $\mathrm{CHO}$ cells stably expressing BGT-1 revealed that NNC05-2090 not only inhibited BGT-1, but also serotonin, noradrenaline, and dopamine transporters, using a substrate uptake assay in $\mathrm{CHO}$ cells stably expressing each transporter, with $\mathrm{IC}_{50}: 5.29,7.91$, and $4.08 \mu \mathrm{M}$, respectively. These values were similar to the $\mathrm{IC}_{50}$ value at BGT-1 $(10.6 \mu \mathrm{M})$. These results suggest that the antiallodynic action of NNC05-2090 is due to the inhibition of both BGT-1 and monoamine transporters.
\end{abstract}

Keywords: neuropathic pain, betaine/GABA transporter, monoamine transporter, NNC05-2090, antiallodynic action

\section{Introduction}

Neuropathic pain is defined as a chronic or persistent pain generated by a pathophysiological alteration in the peripheral and/or central nervous system including spinal dorsal horn neurons. Because this type of pain is refractory against conventional analgesics, the development of a new therapeutic drug is needed. The characteristics

*Correspondence author. sogawa@md.okayama-u.ac.jp Published online in J-STAGE on May 30, 2014

doi: 10.1254/jphs.13146FP of neuropathic pain include an enhanced response to noxious stimuli, thermal and mechanical hyperalgesia, and a pain response to previously non-noxious stimuli, known as allodynia $(1-4)$. Alterations in synaptic transmission within the spinal cord are among the various mechanisms involved in the pathology of neuropathic pain $(5,6)$. Changes in the balance between excitatory and inhibitory synaptic inputs in the spinal dorsal horn may also influence the signal transmission of pain. $\gamma$ Aminobutyric acid (GABA) and glycine serve as the major inhibitory neurotransmitters in the spinal cords of vertebrates. The role of regulating the nociception 
of GABA has been increasingly examined (7). Partial peripheral nerve injuries have been shown to lead to a selective loss in GABA-mediated (GABAergic) inhibition in the superficial dorsal horn of the spinal cord (8). Whether this loss in GABAergic inhibition is due to a decrease in GABA neurons or the loss of GABAsynthesizing enzymes has not yet been elucidated. Maintaining the concentration of GABA in the synaptic cleft mainly depends on the regulation of plasma membrane proteins called GABA transporters (GATs) (9). GATs are members of the $\mathrm{Na}^{+} / \mathrm{Cl}^{-}$dependent transporter family (SLC6 gene family), which also includes the transporters for dopamine, 5-hydroxytriptamine (serotonin: 5-HT), noradrenaline (NA), and amino acids such as glycine, proline, and taurine $(10,11)$. To date, four different GAT members have been isolated from rodents and humans. The nomenclature used for rat and human GATs denotes GAT-1, betaine/GABA transporter (BGT)-1, GAT-2, and GAT-3 (12, 13), whereas the corresponding transporters in mice are termed GAT1 GAT4 (14). The human and rat nomenclature was used in this present study, unless otherwise noted.

GATs may be an attractive target for the treatment of CNS disorders associated with the GABAergic system, such as epilepsy and neuropathic pain $(15,16)$. GAT-1 is widely distributed throughout the entire brain (17) and is primarily found on presynaptic neurons in the synapse $(18,19)$. The localization of GAT-3 was shown to be more restricted than that of GAT-1, and GAT-3 was predominantly expressed on distal astrocytic processes in direct contact with GABAergic neurons $(17,20)$. GAT-2 expression is very limited in the brain and GAT- 2 has been found in multiple peripheral organs $(14,18,21)$. BGT-1 has also been found in multiple peripheral organs, and is localized in the hippocampus and cortex, but not close to GABAergic synapses, and in extrasynaptic regions containing a large amount of astrocytes $(22,23,24)$ which could indicate a specialized pharmacological action different from the other GATs, GAT-1, and GAT-3, which are located in the nerve endings or astrocytes neighboring synapses (18). Recent studies demonstrated the functional role of BGT-1 in the control of neuronal excitability and suggested a possible use of BGT-1 inhibitors for the treatment of epilepsy $(14,25)$. BGT-1 is responsible for the cellular accumulation of betaine and has been proposed to play a role in osmoregulation in the brain (26).

The present study was undertaken to examine whether BGT-1 inhibitors effectively regulated nociceptive responses in neuropathic pain models. We focused on 1-(3-(9H-carbazol-9-yl)-1-propyl)-4-(2-methyoxyphenyl)4-piperidinol (NNC05-2090), an inhibitor that displays moderate selectivity to BGT-1. We investigated neuro- pathic pain-like behavior changes after the administration of NNC05-2090 in a mouse model of sciatic nerve ligation. We also examined behavior changes after the administration of the selective GAT-1 inhibitor 1-(4,4-diphenyl-3-butenyl)-3-piperidinecarboxylic acid (SKF89976A) and selective GAT-3 inhibitor 1-[2-[tris(4methoxyphenyl)methoxy] ethyl]-(S)-3-piperidinecarboxylic acid [(S)-SNAP5114], and we compared these changes with those induced by NNC05-2090.

\section{Materials and Methods}

\section{Chemicals}

NNC05-2090 hydrochloride, SKF89976A hydrochloride, and (S)-SNAP5114 were purchased from Tocris Bioscience (Bristol, UK), and amitriptyline hydrochloride was from Sigma-Aldrich (St. Louis, MO, USA). $\left[{ }^{3} \mathrm{H}\right]$ GABA $(1.295 \mathrm{TBq} / \mathrm{mmol}),\left[{ }^{3} \mathrm{H}\right] 5-\mathrm{HT}$ (1.02 TBq/mmol), $\left[{ }^{3} \mathrm{H}\right] \mathrm{NA}(0.548 \mathrm{TBq} / \mathrm{mmol})$, and $\left[{ }^{3} \mathrm{H}\right]$ dopamine $(1.12 \mathrm{TBq} / \mathrm{mmol})$ were purchased from Perkin-Elmer Life Sciences (Boston, MA, USA). Other reagents used were RibatraAce, KOD-Plus DNA polymerase from TOYOBO (Tokyo), TRIzol Reagent (Invitrogen, Carlsbad, CA, USA), and DNase I (Takara, Shiga).

\section{Animals}

The present study was approved by the Committee of Research Facilities for Laboratory Animal Science in Okayama University, and performed according to both the Guiding Principles for the Care and Use of Laboratory Animals approved by the Japanese Pharmacological Society and the guidelines of Okayama University (Okayama). We used 5-week-old ddY male mice (SLC, Shizuoka), weighing $25-30 \mathrm{~g}$ at the beginning of the study. Mice were housed at $22^{\circ} \mathrm{C} \pm 2{ }^{\circ} \mathrm{C}$ with free access to commercial food and tap water.

\section{Partial sciatic nerve ligation (PSL) model}

Mice were anesthetized with pentobarbital $(60 \mathrm{mg} / \mathrm{kg}$, i.p.). Partial ligation of the sciatic nerve was performed by tying the distal one third to one half of the sciatic nerve, according to the procedure described in rats by Seltzer et al. (27) and adapted to mice by Malmeberg and Basbaum (28). In brief, the sciatic nerve was exposed at the high thigh level through a small incision and was carefully cleared of surrounding connective tissue just distal to the bony prominence of the femur. An 8-0 silk suture was inserted into the nerve with a three-eighthscurved, reversed-cutting mini-needle, tightly ligated so that the dorsal one-third to one-half of the nerve thickness was held within the ligature, and the wound was then closed. In sham-operated mice, the nerve was exposed using the same procedure without ligation. 
We evaluated mechanical allodynia 2 or 3 days after surgery to confirm that animals had developed allodynia and also to remove animals that had not responded adequately. Mechanical allodynia was maintained for at least one month.

\section{Measurement of touch-evoked tactile allodynia}

Tactile allodynia was evaluated by measuring the paw withdrawal threshold in response to probing with a series of calibrated fine filaments (von Frey filaments). Mice were acclimated in individual clear Plexiglas boxes $(9 \times 7 \times 11 \mathrm{~cm})$ on an elevated wire mesh platform to allow access to the planter surface of the injured hindpaws. The von Frey filament was pressed perpendicularly against the mid-planter surface of the hind paw from below the mesh floor and held for 3 to $5 \mathrm{~s}$ with it slightly buckled. The smallest filament that caused the animal to flinch or move the paw away from the stimulus 5 times out of 5 trials at intervals of $5 \mathrm{~s}$ was determined to be the mechanical threshold (29). Values were the average of the threshold at each time point. Tactile allodynia was assessed in some experiments by lightly stroking the injured leg with a paintbrush. Each mouse was placed in an individual plastic cage with wood chips on the floor and subsequently observed. Brushing was performed twice on each side from a backward direction. The tactile allodynia response was ranked according to Minami et al. (30): 0, no response; 1, mild squeaking with attempts to move away from the stroking probe; and 2, vigorous squeaking, biting the stroking probe, and strong efforts to escape from the stroking probe.

\section{Drug preparation and administration}

Animals were used in experiments approximately 2 weeks $(11-16$ days) following nerve ligation. Animals were administered NNC05-2090, SKF89976A, (S)SNAP5114, or amitriptyline. NNC05-2090, SKF89976A, and $(S)$-SNAP5114 were dissolved in dimethyl sulfoxide (DMSO) and diluted appropriately with ACSF or saline (the final concentration of DMSO was less than $0.5 \%$ ). The composition of ACSF (in $\mathrm{mM}$ ) was $142 \mathrm{mM} \mathrm{NaCl}$, $5 \mathrm{mM} \mathrm{KCl}, 2 \mathrm{mM} \mathrm{CaCl} 2,2 \mathrm{mM} \mathrm{MgCl}, 1.25 \mathrm{mM}$ $\mathrm{NaH}_{2} \mathrm{PO}_{4}, 10 \mathrm{mM}$ D-glucose, $10 \mathrm{mM}$ HEPES, and $0.05 \%$ fatty acid-free bovine serum albumin $(\mathrm{pH}$ 7.4). The intraperitoneal (i.p.) injection of drugs was administered in a volume of $0.1 \mathrm{ml} / 10 \mathrm{~g}$ body weight. When given intravenously (i.v.), solutions were injected into the tail vein in a volume of $0.1 \mathrm{ml} / 10 \mathrm{~g}$ body weight. The head of a mouse was placed into a plastic cap and the body was held with one hand for an intrathecal (i.t.) injection. A 27-gauge needle attached to a Hamilton microsyringe was inserted into the subarachnoid space between the L5 and L6 vertebrae of the conscious mouse and $5 \mu$ l of the drug solution was slowly injected, as described by Hylden and Wilcox (31). Accurate placement of the needle was confirmed by a quick "flick" of the mouse's tail, as described by Honda et al. (32).

\section{Analysis of mRNA expression}

The expression of each GAT subtype was analyzed by reverse transcriptase (RT)-polymerase chain reaction (PCR) using the primers specific for each subtype. Total RNA was extracted from the brain cortex, brainstem, cerebellum, and spinal cord using TRIzol Reagent and treated with DNase I (33). First strand cDNA from $1 \mu \mathrm{g}$ total RNA was synthesized with random hexamer primers and $\mathrm{RNaseH}(-)$-MMLV reverse transcriptase (RivatraAce). PCR was performed by initial denaturation at $94^{\circ} \mathrm{C}$ for $2 \mathrm{~min}$, followed by 30 cycles (GAT-1 and GAT-3) or 35 cycles (BGT-1) of $94^{\circ} \mathrm{C}$ for $30 \mathrm{~s}, 58^{\circ} \mathrm{C}$ for $30 \mathrm{~s}$, and $68^{\circ} \mathrm{C}$ for 2 min with a final extension at $68^{\circ} \mathrm{C}$ for 5 min using Kod-Plus OD. The resulting PCR products were analyzed by electrophoresis on $1.5 \%$ agarose gel. Amplicons were isolated from agarose gel, and were then directly sequenced using PCR primers to confirm their sequences (34).

\section{Cell culture and expression}

Chinese hamster ovary $(\mathrm{CHO})$ cells were cultured at $37^{\circ} \mathrm{C}$ under $5 \% \mathrm{CO}_{2} / 95 \%$ air in Minimum Essential Medium-alpha ( $\alpha$-MEM) supplemented with $10 \%$ fetal bovine serum, 100 units $/ \mathrm{ml}$ penicillin G, $100 \mu \mathrm{g} / \mathrm{ml}$ streptomycin, and $0.25 \mu \mathrm{g} / \mathrm{ml}$ fungisone. Cell lines stably expressing the transporters were designated as $\mathrm{CHO} /$ mouse GAT-1, CHO/mouse GAT-2, CHO/mouse GAT-3, $\mathrm{CHO} /$ mouse BGT-1, $\mathrm{CHO} /$ rat noradrenaline transporter (NET), CHO/rat serotonin transporter (SERT), and $\mathrm{CHO} /$ rat dopamine transporter (DAT), respectively, and had been cloned previously $(35-38)$. Cells were diluted in the culture medium, plated in 48 -well culture plates, and cultured for $24 \mathrm{~h}$.

\section{Uptake assay}

Cells were washed 3 times with oxygenated Krebs Ringer HEPES-buffered solution (KRH; $125 \mathrm{mM} \mathrm{NaCl}$, $5.2 \mathrm{mM} \mathrm{KCl}, 1.2 \mathrm{mM} \mathrm{CaCl}_{2}, 1.4 \mathrm{mM} \mathrm{MgSO}_{4}, 1.2 \mathrm{mM}$ $\mathrm{KH}_{2} \mathrm{PO}_{4}, 5 \mathrm{mM}$ glucose, and $20 \mathrm{mM}$ HEPES, $\mathrm{pH}$ 7.3) and incubated for $10 \mathrm{~min}$ at $37^{\circ} \mathrm{C}$ with $10 \mathrm{nM}\left[{ }^{3} \mathrm{H}\right] \mathrm{GABA}$ or other radiolabeled ligands, as described previously $(39,40)$. Aminooxyacetic acid $(100 \mu \mathrm{M})$ and ascorbic acid $(100 \mu \mathrm{M})$ plus pargyline $(50 \mu \mathrm{M})$ were added to the incubation solution during GABA and monoamine (5-HT, NA, and dopamine) uptake assays, respectively. After the removal of excess radioligands by aspiration, the cells were washed rapidly 3 times with ice-cold $\mathrm{KRH}$, and any radioactivity remaining in the cells was 
extracted with $\mathrm{NaOH}(1 \mathrm{M})$ and measured by liquid scintillation spectrometry. Nonspecific uptake was determined in the presence of $10 \mathrm{mM}$ cold GABA for GABA uptake or $10 \mu \mathrm{M}$ cocaine for each monoamine uptake. The inhibitory potency of GAT inhibitors ( $\mathrm{IC}_{50}$ values) was analyzed using Prism 5 (GraphPad Software, San Diego, CA, USA).

\section{Forced-swimming test}

This test was performed using the method described by Porsolt et al. (41). Mice were placed individually into a cylinder (height: $25 \mathrm{~cm}$, diameter $10 \mathrm{~cm}$ ) containing $15 \mathrm{~cm}$ water at $23^{\circ} \mathrm{C}-24^{\circ} \mathrm{C}$. Animals were left to swim in the water for $10 \mathrm{~min}$ followed by gentle drying and returned to their cages. After the pre-session, animals received either vehicle or test samples $30 \mathrm{~min}$ or $1 \mathrm{~h}$ prior to the second swimming exposure $24 \mathrm{~h}$ later. In the second exposure, mice were allowed to swim for $6 \mathrm{~min}$. Because immobility was rarely observed during the first 2 min, only that occurring during the last 4 min was counted. Immobility was considered as the complete absence of active escape-oriented behaviors such as swimming, diving, and exploratory behavior.

\section{Statistical analysis}

Values are expressed as the mean \pm S.E.M. A one-way analysis of variance (ANOVA) was used for multiple comparison followed by Dunnett's test or Bonferroni's test, where appropriate. In some experiments, the results were analyzed by the Student's $t$-test. Significance was defined where $P$-values were less than 0.05 . All statistical analyses were performed with Prism 5 (GraphPad Software, San Diego, CA, USA).

\section{Results}

Effects of GAT inhibitors on mechanical allodynia in a mouse model of PSL

The mechanical withdrawal threshold was significantly lower on the ipsilateral side in partial sciatic nerve ligation (PSL) model mice than in sham-operated animals (Fig. 1). A reduction was not observed in the mechanical withdrawal threshold on the contralateral side (Figs. 2B, 3B, 4, and 5). Mechanical allodynia in the mouse model of PSL was not affected by the saline treatments (Fig. 1B). An increase was observed in the mechanical withdrawal threshold in PSL model mice $3 \mathrm{~h}$ after the administration i.p. of $0.1 \mathrm{mg} / \mathrm{kg}$ NNC05-2090 (Fig. 1B). On the other hand, a $0.3 \mathrm{mg} / \mathrm{kg}$, i.p. injection of SKF89976A did not induce any significant effect on the withdrawal threshold in PSL model mice (Fig. 1B). Intravenous (i.v.) injection of the BGT-1 inhibitor NNC05-2090 significantly reversed mechanical allodynia in PSL model mice (Fig. 2). A dose-dependent response to NNC05-2090 was shown in the range of 0.01$0.1 \mathrm{mg} / \mathrm{kg}$ by an i.v. injection. i.t. injection of the BGT-1 inhibitor NNC05-2090 also significantly reversed mechanical allodynia in PSL model mice, and a dosedependent response to NNC05-2090 was also shown in the range of $15-150$ pmoles by an i.t. injection to PSL model mice (Fig. 3). Antiallodynic effects peaked within $1 \mathrm{~h}$ after the injection of NNC05-2090, with the exception of the $0.01 \mathrm{mg} / \mathrm{kg}$, i.v. administration (Figs. 2 and 3). No significant difference was observed in the withdrawal threshold on the contralateral side after the administration of NNC05-2090. SKF89976A produced a weak antiallodynic response when administered
A

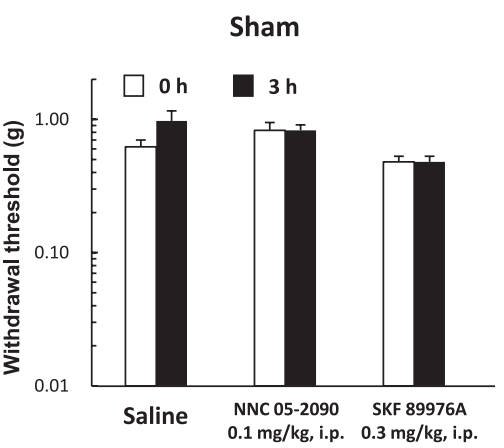

B

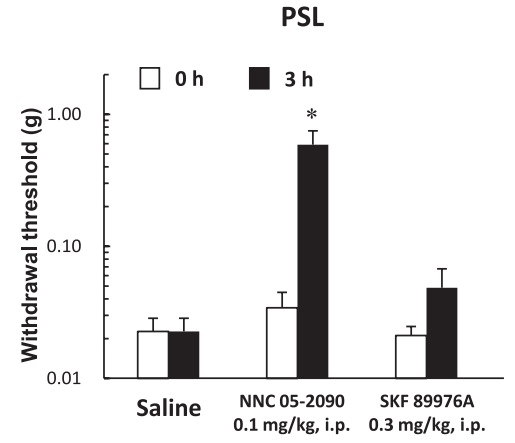

Fig. 1. Effects of the intraperitoneal administration of NNC05-2090 and SKF89976A on mechanical allodynia in a mouse model of partial sciatic nerve ligation. A total of $0.1 \mathrm{mg} / \mathrm{kg} \mathrm{NNC05-2090} \mathrm{and} 0.3 \mathrm{mg} / \mathrm{kg}$ SKF89976A were administered intraperitoneally (i.p.) at time 0,14 days after surgery. Mechanical allodynia was assessed by measuring the withdrawal threshold in response to probing with von Frey hairs in the side of the nerve-injured paw at time 0 and $3 \mathrm{~h}$ after the administration of drugs. NNC05-2090 and SKF89976A were also administrated to sham-operated mice. Data are expressed as the mean \pm S.E.M. $\mathrm{n}=5-9$ mice per group. Control mice received injections with saline. The development of mechanical allodynia after a partial sciatic nerve ligation injury in mice was not affected by the saline treatments. $* P<0.01$, significantly different from the corresponding control values just before the injection (time $0 \mathrm{~h}$ ) of NNC05-2090 or SKF89976A, as determined by the Student's $t$-test. 
i.v. $(0.3 \mathrm{mg} / \mathrm{kg})$ (Fig. 4A). As is shown in Fig. 5A, the i.t. injection of SKF89976A dose-dependently ameliorated the reduction in the withdrawal threshold in PSL model mice. We also injected the GAT-3 inhibitor (S)-SNAP5114 to examine its antiallodynic effect on mechanical allodynia in PSL mice. Neither the i.v. nor i.t. injection of $(S)$-SNAP5114 had a significant effect on the reduction in the withdrawal threshold in PSL model mice (Fig. 4B and 5B).

\section{Comparison of the inhibitory potency of GAT inhibitors}

To analyze the effects of GAT inhibitors on the uptake

\section{A}

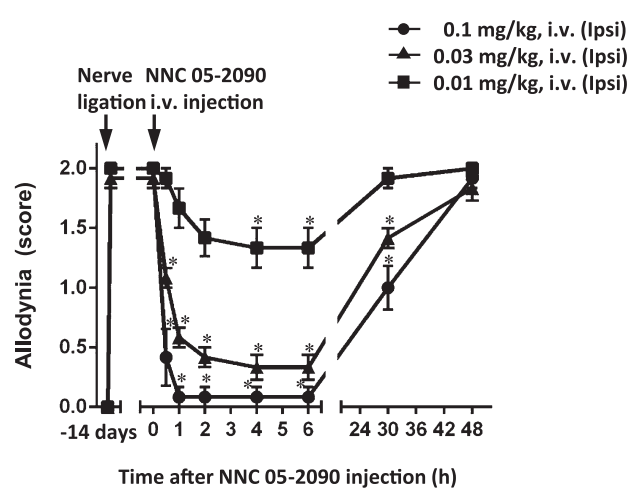

B

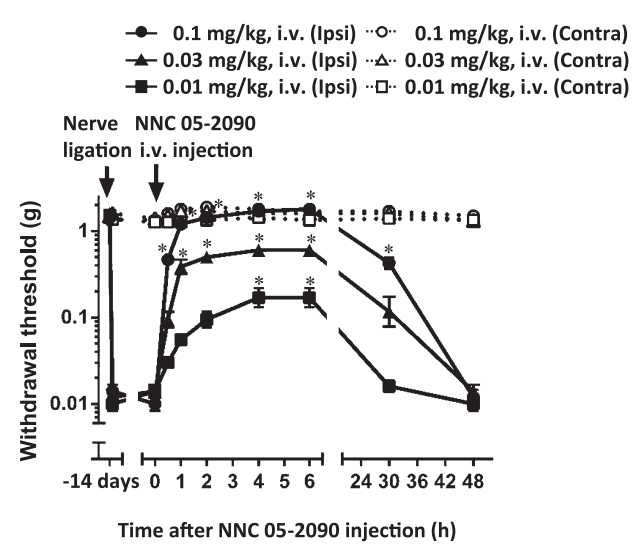

Fig. 2. Effects of the intravenous administration of NNC05-2090 on mechanical allodynia in a mouse model of partial sciatic nerve ligation. Mechanical allodynia was assessed by lightly stroking the flank of each mouse with a paintbrush on the side of the nerve-injured paw (A). Each point represents the average score evaluated at each time point. Mechanical allodynia was measured using the paw withdrawal threshold in response to probing with von Frey hairs (B). NNC05-2090 was administrated intravenously at time 0. Data are expressed as the mean \pm S.E.M. $\mathrm{n}=6$ mice per group; ipsi: the side of the nerve-injured paw, contra: the contralateral side. ${ }^{*} P<0.01$, significantly different from the corresponding control values just before the injection of NNC05-2090, as determined by a one-way ANOVA followed by Dunnett's test. of $\left[{ }^{3} \mathrm{H}\right] \mathrm{GABA}$, we previously established cell lines stably expressing GAT subtypes using CHO cells (38). Using these cell lines together with CHO cells stably expressing rat monoamine transporters SERT, NET, and DAT (37) to compare the potencies in inhibiting GAT subtypes, we analyzed the effect of GAT inhibitors on the uptake of GABA and monoamines. The inhibitors SKF89976A and $(S)$-SNAP5114 exhibited subtype selectivity for GAT-1 $\left(\mathrm{IC}_{50}: 0.28 \mu \mathrm{M}\right)$ and GAT-3 ( $\left.\mathrm{IC}_{50}: 5.31 \mu \mathrm{M}\right)$, respectively (Table 1 ). However, NNC05-2090 potently
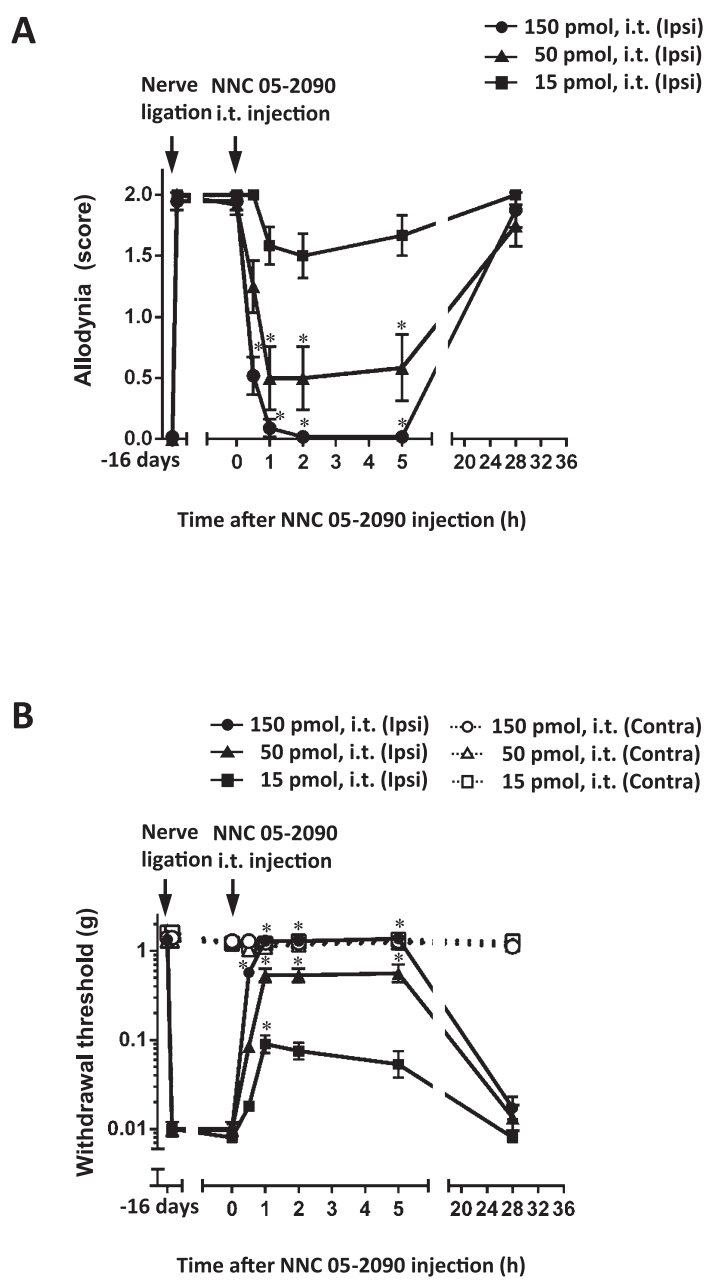

Fig. 3. Effects of the intrathecal administration of NNC05-2090 on mechanical allodynia in a mouse model of partial sciatic nerve ligation. Mechanical allodynia was assessed by lightly stroking the flank of each mouse with a paintbrush on the side of the nerve-injured paw (A). Each point represents the average score evaluated at each time point. Mechanical allodynia was measured using the paw withdrawal threshold in response to probing with von Frey hairs (B). NNC05-2090 was administrated intrathecally at time 0. Data are expressed as the mean \pm S.E.M. $\mathrm{n}=6$ or 7 mice per group; ipsi: the side of the nerve-injured paw, contra: the contralateral side. ${ }^{*} P<0.01$, significantly different from the corresponding control values just before the injection of NNC05-2090, as determined by a one-way ANOVA followed by Dunnett's test. 


\begin{tabular}{|c|c|c|}
\hline$\neg \quad 0.3 \mathrm{mg} / \mathrm{kg}$, i.v. (Ipsi) & & $0.3 \mathrm{mg} / \mathrm{kg}$, i.v. (Contra) \\
\hline$\rightarrow 0.1 \mathrm{mg} / \mathrm{kg}$, i.v. (Ipsi) & .... & 0.1 mg/kg, i.v. (Contra) \\
\hline$-0.03 \mathrm{mg} / \mathrm{kg}$, i.v. (Ipsi) &.$\Delta \cdot$ & $0.03 \mathrm{mg} / \mathrm{kg}$, i.v. (Contra) \\
\hline$-0.01 \mathrm{mg} / \mathrm{kg}$, i.v. (Ipsi) & ..-. & $0.01 \mathrm{mg} / \mathrm{kg}$, i.v. (Contra) \\
\hline
\end{tabular}

A

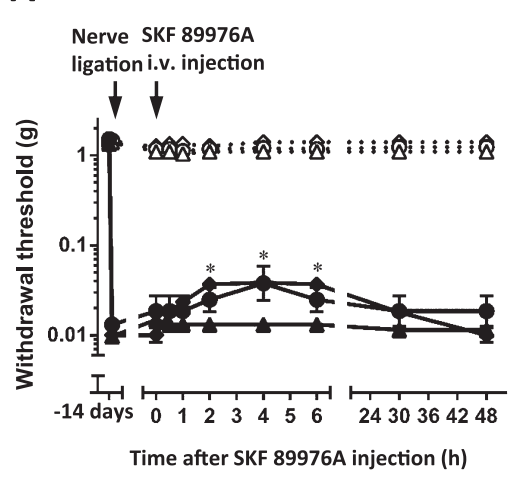

B

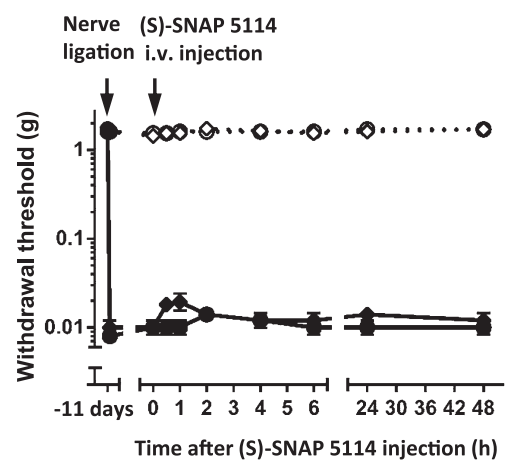

Fig. 4. Effects of intravenous administration of SKF89976A and (S)-SNAP5114 on mechanical allodynia in a mouse model of partial sciatic nerve ligation. SKF89976A (A) or (S)-SNAP5114 (B) were administered intravenously at time 0 . Data are expressed as the mean \pm S.E.M. $\mathrm{n}=6$ or 7 mice per group; ipsi: the side of the nerve injured paw, contra: the contralateral side. ${ }^{*} P<0.01$, significantly different from the corresponding control values just before the injection of SKF89976A (A), or (S)-SNAP5114 (B), as determined by a one-way ANOVA followed by Dunnett's test.

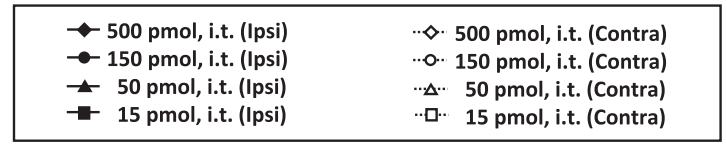

A

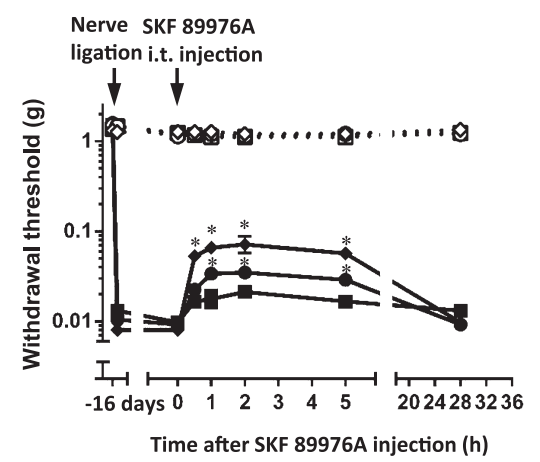

B

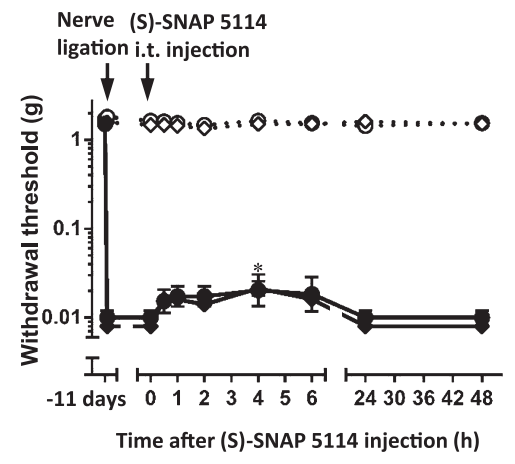

Fig. 5. Effects of intrathecal administration of SKF89976A and (S)-SNAP5114 on mechanical allodynia in a mouse model of partial sciatic nerve ligation. SKF89976A (A) or (S)-SNAP5114 (B) were administered intrathecally at time 0 . Data are expressed as the mean \pm S.E.M. $\mathrm{n}=6-10$ mice per group; ipsi: the side of the nerve injured paw, contra: the contralateral side. ${ }^{*} P<0.01$, significantly different from the corresponding control values just before the injection of SKF89976A (A) or (S)-SNAP5114 (B), as determined by a one-way ANOVA followed by Dunnett's test. inhibited GAT-1 $\left(\mathrm{IC}_{50}: 29.62 \mu \mathrm{M}\right)$, with $\mathrm{IC}_{50}$ values more than 2.5-fold lower being observed for BGT-1 $(10.60 \mu \mathrm{M})$. NNC05-2090 also showed markedly higher $\mathrm{IC}_{50}$ values for GAT-2 $(45.29 \mu \mathrm{M})$ and GAT-3 (22.51 $\mu \mathrm{M})$. These results indicate that of all the GAT inhibitors examined, NNC05-2090 has highest potency inhibiting BGT-1 between GAT inhibitors. NNC05-2090 also inhibited SERT, NET, and DAT ( $\mathrm{IC}_{50}: 5.29 \mu \mathrm{M}, 7.91$ $\mu \mathrm{M}$, and $4.08 \mu \mathrm{M}$, respectively), and these $\mathrm{IC}_{50}$ values were similar to that for BGT-1.

\section{Analysis of behavioral aspects of NNC05-2090 using the forced-swimming test}

The standard antidepressant drug, amitriptyline induced a reduction in the immobility time in mice at a dose of $20 \mathrm{mg} / \mathrm{kg}$, i.p. (Table 2). NNC05-2090 did not induce a reduction in the immobility time in mice at a dose of $0.1 \mathrm{mg} / \mathrm{kg}$, i.p., which was previously shown to have an antiallodynic effect on the mouse model of PSL. However, $2 \mathrm{mg} / \mathrm{kg}$, i.p. administration of NNC052090 reduced the immobility time from $175.7 \pm 21.1$ (vehicle group) to $61.2 \pm 19.6$ (NNC05-2090 treated group) (Table 2).

\section{Discussion}

We investigated whether NNC05-2090, a BGT-1 inhibitor, effectively regulated the nociceptive response in a neuropathic pain model (PSL model) in the present study and also assessed antinociceptive effects after the administration of selective GAT- 1 and GAT-3 inhibitors to determine the participation of BGT-1. The systemic 
Table 1. The inhibitory action of GABA transporter (GAT) inhibitors on GAT subtypes and monoamine transporters

\begin{tabular}{lccc}
\hline Transporters & NNC05-2090 IC $_{50}(\mu \mathrm{M})$ & SKF89976A IC $_{50}(\mu \mathrm{M})$ & $(S)$-SNAP5114 IC $50(\mu \mathrm{M})$ \\
\hline GAT-1 & 29.62 & 0.28 & 446.75 \\
GAT-2 & 45.29 & 137.34 & 48.36 \\
GAT-3 & 22.51 & 302.80 & 5.31 \\
BGT-1 & 10.60 & 481.05 & 132.60 \\
SERT & $5.29 \pm 1.30$ & $3514.00 \pm 866.00$ & $871.07 \pm 151.36$ \\
NET & $7.91 \pm 1.55$ & $202.13 \pm 19.91$ & $1850.67 \pm 361.34$ \\
DAT & $4.08 \pm 0.24$ & $728.80 \pm 48.81$ & $1737.63 \pm 528.88$ \\
\hline
\end{tabular}

CHO cells stably expressing the mouse GAT subtypes, rat serotonin transporter (SERT), rat noradrenaline transporter (NET), and rat dopamine transporter (DAT) were incubated with $10 \mathrm{nM}$ tritium-labeled GABA or monoamines for 10 min in the absence or presence of various concentrations of the GAT inhibitors tested. Values presented for SERT, NET, and DAT are the mean \pm S.E.M. for 3 experiments, with each being performed in duplicate. Data for GAT-1, GAT-2, GAT-3, and BGT-1 represent $\mathrm{IC}_{50}$ values from 2 experiments, with each being performed in duplicate.

Table 2. Effects of amitriptyline and NNC05-2090 on the duration of immobility in mice during the forced-swimming test

\begin{tabular}{lccc}
\hline Drugs & Doses $(\mathrm{mg} / \mathrm{kg}, \mathrm{i.p.})$ & No. of mice per experiment & Duration of immobility $(\mathrm{s})$ \\
\hline Saline & - & 14 & $177.7 \pm 11.8$ \\
Amitriptyline & 20 & 13 & $110.1 \pm 18.3^{*}$ \\
Vehicle & - & 10 & $175.7 \pm 21.1$ \\
NNC 05-2090 & 0.1 & 10 & $153.9 \pm 19.6$ \\
NNC 05-2090 & 2 & 10 & $61.2 \pm 19.6^{\#}$ \\
\hline
\end{tabular}

Saline and amitriptyline were administered $30 \mathrm{~min}$ before the test. Vehicle $(0.5 \%$ DMSO) and NNC 05-2090 were administered $1 \mathrm{~h}$ before the test. The duration of immobility was recorded during the last $4 \mathrm{~min}$ of the 6-min test. Values are expressed as the mean \pm S.E.M. The group treated with amitriptyline was significantly different from the saline group $(* P<0.01)$. The groups treated by NNC 05-2090 were significantly different from the vehicle group $\left({ }^{\sharp} P<0.01\right.$, one-way ANOVA followed by Bonferroni's multiple comparisons test).

administration of NNC05-2090 (both i.p, and i.v. administration routes) induced marked antiallodynic effects in PSL model mice. Furthermore, the i.t. administration of NNC05-2090 showed maximum efficacy. This antiallodynic effect undoubtedly contributed to the effects of the systemic administration of NNC05-2090. NNC05-2090 exhibited a preferential inhibitory effect on BGT-1 over the other GATs. Thomsen et al. (42) reported that NNC05-2090 was a potent inhibitor of BGT-1, the selectivity of which was at least 10-fold stronger than the other GATs. The present results are in line with previous data. On the other hand, SKF-89976A was a highly potent inhibiter of GAT-1, the selectivity of which was 500 -fold stronger than the other GATs. We concluded that the inhibitory effects of NNC 05-2090 on BGT-1 were more selective than those of the other GATs. NNC05-2090 also exhibited $\alpha_{1}$ - and $\mathrm{D}_{2}$-receptor affinities of 266 and $1632 \mathrm{nM}$, respectively (43). Previous studies reported the anticonvulsant effects of NNC05-2090 in rodent seizure models (43). They also reported that the inhibition of GAT through non-GAT-1 transporters induced anticonvulsant effects that differed from those of selective GAT-1 inhibitors (e.g., tiagabine). They suggested that the anticonvulsant effects of NNC052090 were primarily due to the inhibition of GAT-3mediated GABA transport with the potentially minor contribution of the inhibition of BGT-1-mediated GABA transport. However, we demonstrated that a selective GAT-3 inhibitor [(S)-SNAP5114] did not display an antiallodynic effect in the present study. We examined the expressions of BGT-1, GAT-1, and GAT-3 mRNA in the cerebral cortex, cerebellum, brainstem, and spinal cord 14 days after PSL using RT-PCR. BGT-1, GAT-1, and GAT-3 mRNA were expressed in the cerebral cortex, cerebellum, brainstem, and spinal cord in both shamoperated mice and PSL model mice (data not shown). The GAT-3 inhibitor, $(S)$-SNAP5114, had no marked antiallodynic effect in our PSL model mice, but this could not be attributed to the lack of GAT-3 expression in the CNS during a persistent painful state. Thus, the inhibition of GAT-3-mediated GABA transport by NNC05-2090 may play a minor role in its antiallodynic effect.

On the other hand, we also demonstrated the dose- 
dependent antiallodynic effect of the GAT-1 selective inhibitor SKF89976A, which was only observed via the i.t. administration route. A previous study suggested the antinociceptive effect of another selective GAT-1 inhibitor, NO-711, in sciatic nerve chronic constriction injury model rats (44). Although we could not compare the strength of the antinociceptive action between NO711 with SKF89976A, we speculated that the inhibition of GAT-1 was at least related to the antiallodynic effect on neuropathic pain with nerve injury. However, based on the result that NNC05-2090 had a more pronounced effect than the selective GAT-1 inhibitor (SKF89976A) at a low dose, we suggest that the inhibition of BGT-1 played an important role in the antiallodynic effect of NNC05-2090. Lehre et al. (45) recently reported that BGT-1 knockout mice exhibited normal development and seizure susceptibility indistinguishable from that of wild-type mice in various seizure threshold tests. They concluded that BGT-1 mRNA was present in the brain, but that its levels were several hundred times lower than those of GAT-1 mRNA, possibly explaining the apparent lack of a phenotype. Therefore, we predicted that the expression of BGT-1 may vary in the spinal cord of sciatic nerve-ligated mice exhibiting neuropathic pain. Even if the expression of BGT-1 is low in the spinal cord, its inhibition is primarily responsible for the antinociceptive effects of NNC05-2090 if the expression of BGT-1 increases with neuropathic pain. We showed that BGT-1 mRNA was expressed in the supraspinal and spinal regions in both sham-operated and PSL model mice (data not shown). We could not assess this result in sufficient detail because we did not analyze the expression of each mRNA quantitatively. The functional role of or quantitative change in BGT-1 in a painful state needs to be studied in more detail. BGT-1 has been found in extrasynaptic regions containing a large amount of astrocytes $(23,24)$, which could indicate a specialized pharmacological action different from the other GATs.

We previously reported that antidepressants inhibited GABA uptake through BGT-1 and other GAT subtypes. All the antidepressants tested inhibited uptake in a rank order of potency with BGT-1 > other GAT subtypes (38). Tricyclic antidepressants (TCAs), which are known as the inhibitors of monoamine neurotransmitter transporters, especially SERT and NET transporters (46), are therapeutically useful ligands for the treatment of neuropathic pain (47). These TCAs may act on inhibitory serotonergic and noradrenergic descending pathways in the spinal cord, resulting in the inhibition of pain. NNC05-2090 is a nipecotic acid derivative, and it has a tricyclic structure that is similar to that of TCAs. In the present study, we examined the potency of NNC05-2090 in inhibiting monoamine neurotransmitter transporters including SERT and NET. The results obtained showed that NNC05-2090 also inhibited SERT, NET, and DAT in $\mathrm{CHO}$ cells stably expressing each transporter using a substrate uptake assay, with $\mathrm{IC}_{50}$ values of 5.29, 7.91, and 4.08 , respectively. These $\mathrm{IC}_{50}$ values were similar to that of BGT-1 $(10.60 \mu \mathrm{M})$. On the other hand, the potencies of both SKF89976A and (S)-SNAP5114 were markedly lower than that of NNC05-2090. These results suggest that the inhibition of monoamine transporters may also be one of the causes for the antinociceptive effect of NNC05-2090. The administration of $\mathrm{NNC} 05-2090$ at $2 \mathrm{mg} / \mathrm{kg}$, i.p. induced a reduction in immobility time in mice. The forced-swimming test is commonly used to evaluate antidepressant agents. Although the antinociceptive effect of $0.1 \mathrm{mg} / \mathrm{kg}$, i.p. was observed on PSL induced in the neuropathic pain model in mice, a reduction was not detected in the immobility time by the forced-swimming test. NNC052090 is known to have an antidepressant effect, but this was not observed at the dose that induced an antinociceptive effect. TCAs have been shown to induce antinociceptive effects at doses lower than those used in the treatment of depression (48). These antinociceptive effects appear to be independent of the antidepressant effects of these drugs. Thus, we suggest that the antiallodynic effect of NNC05-2090 is also independent of its antidepressant effects. The mechanisms underlying the antinociceptive effects of TCAs are complicated. It is assumed that TCAs were, at least in part, inhibited by the blockade of NA and 5-HT reuptake in the synaptic clefts of the spinal cord. As a result, the descending monoaminergic pain inhibitory system was activated in the spinal cord.

Furthermore, antidepressants acting on the noradrenergic or both the noradrenergic and serotonergic systems appear to be more effective than those working on the serotonergic system alone (49). NNC05-2090 inhibits the reuptake of 5-HT and NA in a relatively balanced manner. It also influences the reuptake inhibition of GABA. NNC05-2090, which acts on the GABAergic, noradrenergic, and serotonergic systems, appears to be more effective than those working on the serotonergic system alone or GABAergic system alone (e.g., selective 5-HT reuptake inhibitor and selective GAT-1 inhibitor). We did not examine the relationship between the spinal action of NNC05-2090 and spinal levels of NA, 5-HT, and GABA in detail. Although many questions remain to be answered, the results of the present study suggest that NNC05-2090 may be applicable to the treatment of neuropathic pain. Further pharmacological and morphological investigations are needed to determine the central site of the antiallodynic action of NNC052090 and other BGT-1 inhibitors at the spinal or supra- 
spinal level in each model of chronic neuropathic pain.

In conclusion, the results of the current study showed that NNC05-2090 had an antiallodynic effect on mechanical allodynia in a mouse model of neuropathic pain. Furthermore, although the contribution of GAT-1 and adrenergic agonistic effects cannot be entirely ruled out, the antiallodynic action of NNC05-2090 was probably attributed to the inhibition of both BGT-1 and monoamine transporters including SERT and NET.

\section{Acknowledgments}

This study was supported in part by a Grant-in-Aid for Scientific Research from the Ministry of Education, Culture, Sports, Science and Technology, Japan. Experiments were carried out using equipment at the RI Research Center of Okayama University Dental School.

\section{References}

1 Courteix C, Eschalier A, Lavarenne J. Streptozotocin-induced diabetic rats: behavioural evidence for a model of chronic pain. Pain. 1993;543:81-88.

2 Elliott KJ. Taxonomy and mechanisms of neuropathic pain. Semin Neurol. 1994;14:195-205.

3 Woolf CJ, Doubell TP. The pathophysiology of chronic painincreased sensitivity to low threshold A beta-fibre inputs. Curr Opin Neurobiol. 1994;4:523-534.

4 Calcut NA, Jorge MC, Yaksh TL, Chaplan SR. Tactile allodynia and formalin hyperalgesia in streptozotocin-diabetic rats: effects of insulin, aldose reductase inhibition and lidocaine. Pain. 1996; 68:293-299.

5 Zimmermann M. Pathobiology of neuropathic pain. Eur J Pharmacol. 2001;429:23-37.

6 Cambell JN, Meyer RA. Mechanisms of neuropathic pain. Neuron. 2006;52:77-92.

7 Evans JE, Frosholm, Rotter A. Embryonic and postnatal expression of four gamma-aminobutyric acid transporter mRNAs in the mouse brain and leptomeninges. J Comp Neuronal. 1996; 376:431-446.

8 Moore KA, Kohno T, Karchewski LA, Scholz J, Baba H, Woolf CJ. Partial peripheral nerve injury promotes a selective loss of GABAergic inhibition in the superficial dorsal horn of the spinal ford. J Neurosci. 2002;22:6724-6731.

9 Iversen LL. Role of transmitter uptake mechanisms in synaptic neurotransmission. Br J Pharmacol. 1971;41:571-591.

10 Gether U, Peter HA, Larsson OM, Shousboe A. Neurotransmitter transporters: molecular function of important drug targets. Trends Pharmacol Sci. 2006;27:375-383.

11 Bröer S, Gether U. The solute carrier 6 family of transporters. British J Pharmacol. 2012;167:256-278.

12 Guastella J, Nelson N, Nelson H, Czyzyk L, Keynan S, Miedel $\mathrm{MC}$, et al. Cloning and expression of a rat brain GABA transporter. Science. 1990;249:13032306.

13 Borden LA, Smith KE, Harting PR, Bramchek TA, Weinshank RL. Molecular heterogeneity of the gamma-aminobutyric acid (GABA) transport system. Cloning of two novel high affinity GABA transporters from rat brain. J Biol Chem. 1992;267: 21098-21104.
14 Liu QR, Lopez-Corcuera B, Mandiyan S, Nelson H, Nelson N. Molecular characterization of four pharmacologically distinct $\gamma$-aminobutyric acid transporters in mouse brain. J Biol Chem. 1993;268:2106-2112.

15 Dalby NO. Inhibition of $\gamma$-aminobutyric acid uptake: Anatomy, physiology and effects against epileptic seizures. Eur J Pharmacol. 2003;479:127-137.

16 Madsen KK, White HS, Schousboe A. Neuronal and nonneuronal GABA transporters as targets for antiepileptic drugs. Pharmacol Ther. 2010;125:394-401.

17 Durkin MM, Smith KE, Borden LA, Weinshank RL, Branchek TA, Gustafson EL. Localization of messenger RNAs encoding three GABA transporters in rat brain: an in situ hybridization study. Brain Res Mol Brain Res. 1995;33:7-21.

18 Borden LA. GABA transporter heterogeneity: Pharmacology and cellular localization. Neurochem Int. 1996;29:335-356.

19 Conti F, Melone M, DeBiasi S, Minelli A, Brecha NC, Ducati A. Neuronal and glial localization of GAT-1, a high-affinity gamma-aminobutyric acid plasma membrane transporter, in human cerebral cortex: with a note on its distribution in monkey cortex. J Comp Neurol. 1998;396:51-63.

20 Minelli A, DeBiase S, Brecha N, Zuccarello LV, Conti F. GAT-3, a high affinity GABA plasma membrane transporter, is localized to astrocytic processes, and it is not confined to the vicinity of GABAergic synapses in the cerebral cortex. J Neurosci. 1996; 16:6255-6264.

21 Conti F, Zuccarello LV, Barbaresi P, Minelli A, Brecha NC, Melone M. Neuronal, glial and epithelial localization of gammaaminobutyric acid transporter 2, a high-affinity gamma-aminobutyric acid plasma membrane transporter, in the cerebral cortex and neighboring structures. J Comp Neurol. 1999;109:482-494.

22 Lopez-Corcuera B, Liu QR, Mandiyan S, Nelson H, Nelson N. Expression of a mouse brain cDNA encoding novel gammaaminobutyric acid transporter. J Biol Chem. 1992;267:1749117493.

23 Borden LA, Smith KE, Gustafson EL, Branchek TA, Weinshank RL. Cloning and expression of a betaine/GABA transporter from human brain. J Neurochem. 1995;64:977-984.

24 Zhu XM, Ong WY. A light and electron microscopic study of betaine/GABA transporter distribution in the monkey cerebral neocortex and hippocampus. J Neurocytol. 2004;33:233-240.

25 Schousboe A, Larsson OM, Sarup A, White, HS. Role of the betaine/GABA transporter (BGT-1/GAT2) for the control of epilepsy. Eur J Pharmacol. 2004;500:281-287.

26 Yamauchi A, Uchida S, Kwon HM, Preston AS, Robey RB, Garcia-Perez A et al. Cloning of a $\mathrm{Na}^{+}-$and $\mathrm{Cl}^{-}$-dependent betaine transporter that is regulated by hypertonicity. J Biol Chem. 1992;267:649-652.

27 Seltzer Z, Dubner R, Shir Y. A novel behavioral model of neuropathic pain disorders produced in rats by partial sciatic nerve injury. Pain. 1990;43:205-218.

28 Malmberg AB, Basbaum AI. Partial sciatic nerve injury in the mouse as a model of neuropathic pain: Behavioral and neuroanatomical correlates. Pain. 1998;76:215-222.

29 Morita K, Monotyama N, Kitayama T, Morioka N, Kifune K, Dohi T. Spinal antiallodynia action of glycine transporter inhibitors in neuropathic pain models in mice. J Pharmacol Exp Ther. 2006;326:633-645.

30 Minami T, Nishibara I, Ito S, Sakamoto K, Hyodo M, Hayaishi O. Nitric oxide mediates allodynia induced by intrathecal 
administration of prostaglandin $\mathrm{E}_{2}$ or prostaglandin $\mathrm{F}_{2 \alpha}$ in conscious mice. Pain. 1995;61:285-290.

31 Hylden JL, Wilcox GL. Intrathecal morphine in mice: a new technique. Eur J Pharmacol. 1980;67:313-316.

32 Honda K, Harada A, Takano Y, Kamiya H. Involvement of M3 muscarinic receptors of the spinal cord in formalin-induced nociception in mice. Brain Res. 2000;859:38-44.

33 Sogawa CA, Sogawa N, Yamamoto T, Oda N, Inoue T, Onodera $\mathrm{K}$, et al. Localization of metallothionein (MT and expression of MT isoforms induced by cadmium in rat dental pulp. Jpn J Pharmacol. 2001;86:65-72.

34 Sogawa C, Mitsuhata C, Kumagai-Morioka K, Sogawa N, Ohyama K, Morita K, et al. Expression and function of variants of human catecholamine transporters lacking the fifth transmembrane region encoded by exon 6. PLos One. 2010;5:e11945.

35 Sato T, Kitayama S, Mitsuhata C, Ikeda T, Morita K, Dohi T. Selective inhibition of monoamine neurotransmitter transporters by synthetic local anesthetics. Naunyn-Schmiedeberg's Arch Pharmacol. 2000;361:214-220.

36 Sogawa C, Sogawa N, Tagawa J, Fujino A, Ohyama K, Asanuma M, et al. 5-Methoxy-N,N-diisopropyltryptamine (Foxy), a selective and high affinity inhibitor of serotonin transporter. Toxicol Lett. 1997;170:75-82.

37 Sogawa C, Sogawa N, Ohyama K, Kkura-Hanajiri R, Goda Y, Sora I, et al. Methylone and monoamine transporters: correlation with toxicity. Curr Neuropharmacol. 2011;9:58-62.

38 Gerile, Sogawa C, Ohyama K, Masuko T, Kusama T, Morita K, et al. Inhibitory action of antidepressants on mouse betaine/ GABA transporter (BGT1) heterologously expressed in cell cultures. Int J Mol Sci. 2012;13:2578-2589.

39 Kitayama S, Shimada S, Xu H, Markham L, Donovan MD, Uhl GR. Dopamine transporter site-directed mutations differentially alter substrate transport and cocaine binding. Proc Natl Acad Sci U S A. 1992;89:7782-7785.

40 Sugimura M, Kitayama S, Morita K, Irifune M, Takarada T,
Kawahara M, Dohi T. Effects of volatile and intravenous anesthetics on the uptake of GABA, glutamate and dopamine by their transporters heterologously expressed in COS cells and in rat brain synaptosomes. Toxicol Lett. 2001;123:69-76.

41 Porsolt RD, Bertin A, Jalfre M. Behavioural despair in mice: a primary screening test for antidepressants. Arch int Pharmacodyn. 1997;229:327-336.

42 Thomsen C, Sørensen PO, Egebjerg J. 1-(3-(9H-Carbazol-9-yl)1-propyl)-4-(2-methyoxyphenyl)-4-piperidinol, a novel subtype selective inhibitor of the mouse type II GABA transporter. Br. J. Pharmacol. 1997;120:983-985.

43 Dalby NO, Thomsen C, Fink-Jensen A, Lundbeck J, Søkilde B, Man CM, et al. Anticonvulsant properties of two GABA uptake inhibitors NNC 05-2045 and NNC 05-2090, not acting preferentially on GAT-1. Epilepsy Res. 1997;28:51-61.

44 Li Y, Li Y, Gu P, Fu B. Liu F, Li E. Analgesic effect of intrathecally g-aminobutyric acid transporter-1 inhibitor NO-711 administrating on neuropathic pain in rats. Neurosci Lett. 2011; 494:6-9.

45 Lehre AC, Rowley NM, Zhou Y, Holmseth C, Guo C, Holen T, et al. Deletion of the betaine-GABA transporter (BGT1; slc6a12) gene does not affect seizure thresholds of adult mice. Epilepsy Res. 2011;95:70-81.

46 Langer ZS, Schoemaker H. Effects of antidepressants on monoamine transporters. Prog Neuropsychopharmacol Biol Psychiat. 1988;12:193-216.

47 Todd AJ. Neuronal circuitry for pain processing in the dorsal horn. Nature Rev Neurosci. 2010;11:823-836.

48 Sansone RA, Sansone LA. Antidepressants and pain management. Psychiatry. 2008;5:16-19.

49 Ikeda T, Ishida Y, Naono R, Takeda R, Abe H, Nakamura T, et al. Effects of intrathecal administration of newer antidepressants on mechanical allodynia in rat models of neuropathic pain. Neurosci Res. 2009;63:42-46. 